\title{
Impact of atmospheric small-scale fluctuations on climate sensitivity
}

\author{
R. Seiffert ${ }^{1,2}$ and J.-S. von Storch ${ }^{2}$ \\ Received 31 January 2008; revised 7 April 2008; accepted 9 April 2008; published 21 May 2008.
}

[1] Climate change scenarios are based on numerical models with finite spatial and temporal resolutions. The impact of unresolved processes is parameterized without taking the variability induced by subscale processes into account. This drawback could lead to an over-/underestimation of the climate sensitivity. The aim of this study is to investigate the impact of small-scale atmospheric fluctuations on the modeled climate sensitivity to increased $\mathrm{CO}_{2}$ concentration. Using a complex coupled atmosphere-ocean general circulation model (ECHAM5/MPI-OM) climate response experiments with enhanced small-scale fluctuations are performed. Our results show that the strength of the global warming due to a $\mathrm{CO}_{2}$ doubling depends on the representation of small-scale fluctuations. Reducing the horizontal diffusion by a factor of 3 leads to an increase of the equilibrium climate sensitivity by $13 \%$. If white noise is added to the small scales, the climate sensitivity tends to weaken. The largest changes in responses occur in the upper troposphere. Citation: Seiffert, R., and J.-S. von Storch (2008), Impact of atmospheric small-scale fluctuations on climate sensitivity, Geophys. Res. Lett., 35, L10704, doi:10.1029/ 2008GL033483.

\section{Introduction}

[2] Estimating climate response to increasing greenhouse gas concentrations is one of the main challenges in numerical climate modelling. However, different climate models show different climate responses although they were forced in the same way. The projected globally averaged surface warming at the end of the 21 st century ranges from $1.7 \mathrm{~K}$ to $4.4 \mathrm{~K}$ in the A1B Scenario of 4th Assessment Report published by the Intergovernmental Panel on Climate Change (IPCC) [2007]. This transient climate response is closely related to the equilibrium climate sensitivity, which is defined as the equilibrium change in global surface temperature due to a doubling of $\mathrm{CO}_{2}$ concentration. This sensitivity is highly uncertain and often estimated by a probability density function [e.g., Murphy et al., 2004; Knutti et al., 2006]. Several nonlinear thermodynamical feedback processes are generally called to account for the great uncertainty of the climate sensitivity. These feedbacks involve interactions of water vapor, clouds, temperature lapse rate and surface albedo with the earth's radiation budget [Bony et al., 2006]. Several studies [e.g., Colman, 2003; Soden and Held, 2006; Webb et al., 2006] have estimated the strength of the feedback processes in different

\footnotetext{
${ }^{1}$ International Max Planck Research School on Earth System Modelling, Hamburg, Germany.

${ }^{2}$ Max Planck Institute for Meteorology, Hamburg, Germany.
}

Copyright 2008 by the American Geophysical Union. 0094-8276/08/2008GL033483 climate models and provided valuable insight into the origins of varying model sensitivities.

[3] Nevertheless, the climate system is not only determined by the interactions of complex thermodynamical processes but also by nonlinear dynamical coupling of various scales of motion. As a consequence of the limited model resolution the governing equations are truncated at a certain scale. Dynamical processes below this scale cannot be adequately represented in the models and have to be parameterized. Such parameterizations generally do not take the variability induced by the subgrid-scale processes into account, even though variations near the truncation scale depend on the model resolution [von Storch, 2004; Seiffert et al., 2006]. Due to the dynamical coupling between small and large scales, small-scale variability can affect the statistics of large-scale variables [von Storch, 2004]. According to the fluctuation dissipation theorem [Leith, 1975], such statistics are crucial for the responses to a changed external forcing (e.g., $\mathrm{CO}_{2}$ increase). Neglecting small-scale variability could have an influence on the model sensitivity to $\mathrm{CO}_{2}$ forcing.

[4] One approach to investigate whether neglecting variability on scales smaller than the truncation scale affects model sensitivities is to increase the model resolution. Kiehl et al. [2006] found that the climate sensitivity of the Community Climate System Model 3 (CCSM3) increases with increased horizontal resolution. The equilibrium climate sensitivity of the high-resolution version (T85) was $17 \%$ higher than the model sensitivity of the low-resolution version (T31). In contrast, the atmospheric general circulation model ECHAM5 of the Max Planck Institute (MPI) for Meteorology coupled to a fixed-depth $(50 \mathrm{~m})$ mixed layer ocean and thermodynamical sea ice module experiences a reversed trend (E. Roeckner, personal communication, 2007). The results of May and Roeckner [2001], who were using the predecessor of the MPI model, ECHAM4, also suggest a weaker climate response to increased $\mathrm{CO}_{2}$ concentration when changing the resolution from T42 to T106. The opposite trends in the two models, CCSM3 and ECHAM, can be ascribed to many factors. The different parameterizations in the models certainly play an important role. Since parameterizations in general depend on the chosen model resolution, it is hard to distinguish between the effect of changed parameterizations due to different resolution and the effect of additional resolved small-scale processes on the climate sensitivity.

[5] The purpose of this paper is to systematically address the question of whether and how small-scale atmospheric fluctuations affect the modeled climate sensitivity to increased $\mathrm{CO}_{2}$ concentration. Using the coupled atmosphereocean general circulation model ECHAM5/MPI-OM we carried out idealized response experiments with enhanced small-scale fluctuations. Two different approaches are used to modify the small-scale fluctuations. These approaches 
Table 1. Overview of the Experiments Carried out With ECHAM5/MPI-OM

\begin{tabular}{lcc}
\hline & $1 \times \mathrm{CO}_{2}$ & $2 \times \mathrm{CO}_{2}$ \\
\hline Control experiments & ctr11x & ctr12x \\
Reduced diffusion $\tau_{0}=24 \mathrm{~h}$ & diffus1x_24 & diffus2x_24 \\
Reduced diffusion $\tau_{0}=36 \mathrm{~h}$ & diffus1x_36 & diffus2x_36 \\
Moderate noise $\sigma_{\text {noise }}=3 \cdot 10^{-2} K$ & noise1x_3 & noise2x_3 \\
$\left(3 \cdot 10^{-7} s^{-1}\right)$ & & \\
High noise $\sigma_{\text {noise }}=6 \cdot 10^{-2} K$ & noise1x_6 & noise2x_6 \\
$\left(6 \cdot 10^{-7} s^{-1}\right)$ & & \\
\hline
\end{tabular}

and the experimental design are described in section 2. Results are presented in section 3. Discussions and conclusions are given in the final section.

\section{Method}

\subsection{Model}

[6] ECHAM5/MPI-OM is a coupled atmosphere-oceansea ice general circulation model developed at the MPI for Meteorology in Hamburg. The model (with different resolutions) has been successfully applied in several research projects [e.g., Jungclaus et al., 2006; Roeckner et al., 2006; IPCC, 2007]. Details of the different model components are given by Roeckner et al. [2003] and Marsland et al. [2003]. For this study we used the low-resolution version (atmosphere: T31 $\left(\approx 3.8^{\circ}\right.$ by $\left.3.8^{\circ}\right)$ and 19 vertical levels, ocean: $\approx$ $3^{\circ}$ by $3^{\circ}$ and 40 vertical levels).

\subsection{Two Approaches to Modify the Small-Scale Fluctuations}

[7] The first method to increase the small-scale fluctuations involves a modification of the horizontal diffusion parameterization scheme of ECHAM5. The horizontal diffusion damps to a large extent the variability of small-scale components and can therefore be used to enhance smallscale variability. This parameterization is applied in spectral space on the prognostic variables: temperature, vorticity and divergence. The horizontal diffusion is mainly used to ensure a realistic energy spectrum of the resolved scales. The time rate of change of the spectral coefficient $X_{l, m}$ caused by the horizontal diffusion is defined as

$$
\left.\frac{\partial X_{l, m}}{\partial t}\right|_{\text {horizontal diffusion }}=-K_{l} X_{l, m}
$$

with

$$
K_{l}=\frac{1}{\tau_{0}}\left(\frac{l(l+1)}{l_{0}\left(l_{0}+1\right)}\right)^{q} .
$$

[8] The damping factor $K_{l}$ depends strongly on the total wavenumber $l . l_{0}$ marks the truncation scale of the model (here: $l_{0}=31$ ). The exponent $q$ depends on the vertical level ranging from 2 in the uppermost three levels to 10 in the middle and lower troposphere. The damping time scale $\tau_{0}$ controls the strength of the diffusion. In the standard lowresolution version it is set to $\tau_{0}=12 \mathrm{~h}$. An increase in $\tau_{0}$ leads to a weaker damping and hence an enhancement of primarily the small-scale variability.
[9] In the second approach the horizontal diffusion parameterization scheme remains unchanged. Instead we add noise to the smallest resolved scales. At each time step white noise is added to the spectral coefficients of temperature, divergence and vorticity with a total wavenumber $\geq 26$. Note that the explicit representation of the small-scale processes related to wavenumbers close to the truncation scale $\left(l_{0}=31\right)$ is not reliable anyway. The noise mimics a possible impact of unresolved processes. However, it does not qualify for a realistic parameterization of the subgridscale variability. Rather, it aims to isolate the impact of enhanced small-scale fluctuations on the climate sensitivity in the framework of idealized experiments. We do not try to improve the representation of subgrid-scale processes by stochastic parameterization.

\subsection{Experiments}

[10] The experiments are carried out in pairs. Each set of experiments comprises one integration done with preindustrial $\mathrm{CO}_{2}$ concentration (280 ppm) and one integration with doubled $\mathrm{CO}_{2}$ concentration. The difference of these two integrations can then be referred to as the response of the system to the increased $\mathrm{CO}_{2}$ concentration. The $2 \times \mathrm{CO}_{2}$ experiment was originally started from a state of the preindustrial integration. The doubled $\mathrm{CO}_{2}$ concentration was achieved by a $1 \%$ per year increase until the final value of $560 \mathrm{ppm}$ was reached. Holding the concentration constant, the model was further integrated for 880 years. The preindustrial integration has a total length of 1500 years. From these long integrations only the last 50 years were used for the analysis. Table 1 gives an overview of the experiments. Experiments $\operatorname{ctrl} 1 \mathrm{x}$ and $\operatorname{ctrl} 2 \mathrm{x}$ correspond to the control integrations of the standard model with no changes of the representation of the small-scale fluctuations. ' $1 \mathrm{x}$ ' and ' $2 \mathrm{x}$ ' denote $1 \times \mathrm{CO}_{2}$ and $2 \times \mathrm{CO}_{2}$. The other experiments are 150 years continuations of $\operatorname{ctrl} 1 \mathrm{x}$ and $\operatorname{ctrl} 2 \mathrm{x}$ in which the model was altered in the way described below. The first 100 years of the continuations are excluded from the analysis. All model runs are sufficiently equilibrated.

[11] In experiments diffus $1 x \_24$, diffus $2 x \_24$, diffus $1 x_{-} 36$ and diffus $2 x_{-} 36$, the horizontal diffusion is reduced. The damping time $\tau_{0}$ is increased by a factor of 2 or 3 from the standard value of $12 \mathrm{~h}$ to $24 \mathrm{~h}$ or $36 \mathrm{~h}$. Furthermore, four experiments with additional noise in the smallest resolved scales are carried out: noise $1 x$ 3, noise 2x_3, noise1x_6 and noise2x_6. '_3' and ' 6' distinguishes between two noise intensities. The standard deviations of the noise $\sigma_{\text {noise }}$ are $3 \times 10^{-2} \mathrm{~K}$ in experiments noise $1 \mathrm{x} \_3$ and noise $2 \mathrm{x} \_3\left(6 \times 10^{-2} \mathrm{~K}\right.$ in experiments noise $1 \mathrm{x}_{-}^{-} 6$ and noise $2 \mathrm{x}_{-} 6$ ) for temperature and $3 \times 10^{-7} s^{-1}\left(\overline{6} \times 10^{-7} s^{-1}\right)$ for vorticity and divergence. These values are roughly 3 and 6 times larger than the values obtained in the experiment ctrllx.

[12] In addition to the experiments described above, we will further use data obtained from a pre-industrial controlexperiment with a higher resolution (atmosphere: T63L31, ocean: $1.5^{\circ} \times 1.5^{\circ}$ ).

\section{Results}

[13] How the additional noise or the reduced horizontal diffusion affect the variability of the system is illustrated in 

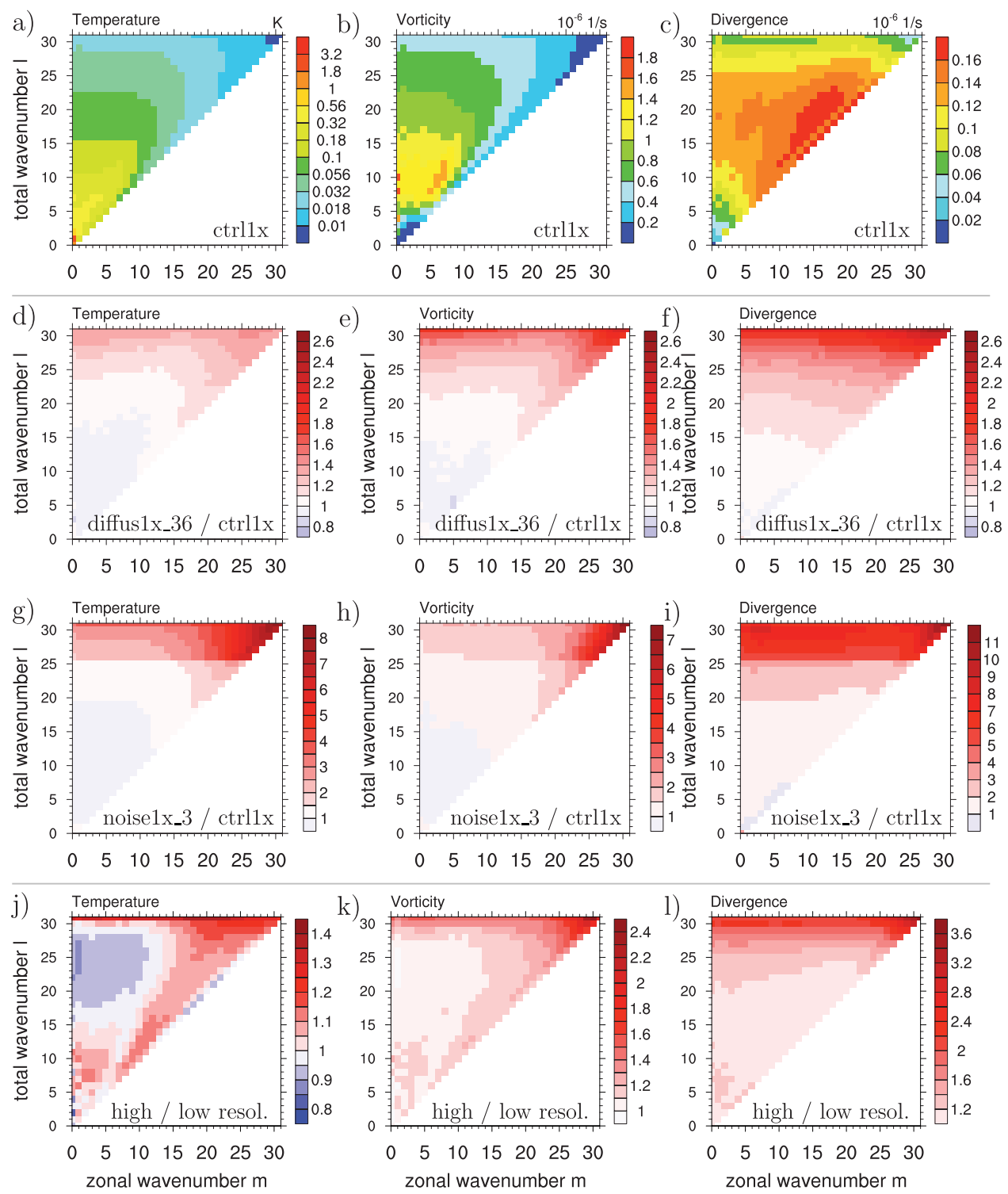

Figure 1. (a)-(c) Standard deviations of spectral coefficients of experiment ctrllx. Ratio of the standard deviations of the spectral coefficients: (d)-(f) diffus1x_3/ctrl1x, (g)-(i) noise1x_36/ctrl1x, and (j)-(1) ctrl1x(T63L31)/ctrl1x(T31L19). All estimates shown here are based on 6-hourly data and 50-year averages at model levels $\approx 500 \mathrm{hPa}$. Please note the different color scales.

Figure 1. Figures $1 \mathrm{a}-1 \mathrm{c}$ show the standard deviations of the spectral coefficients of temperature, vorticity and divergence in ctrllx. Low wavenumbers correspond to largescale components and high wavenumbers to small-scale components. Figures $1 \mathrm{~d}-1 \mathrm{i}$ display the ratio of the standard deviations from experiments with modified small-scale components to the standard deviations obtained from the experiment ctrllx. As expected the variability of spectral coefficients with high total wavenumbers is significantly enhanced if the horizontal diffusion is reduced or if noise is added to the small-scale components. (The other two experiments diffus1x_24 and noise1x_6, which are not shown, show qualitative the same behavior.) Note that the much larger gain of small-scale variability in noise $1 \mathrm{x} 3$ compared to diffus $1 \mathrm{x} 36$ is due to the rather high noise intensity used. By decreasing the noise intensity it would be possible to induce changes of similar magnitude.
[14] How well do our idealized experiments compare to the situation if the model resolution is actually increased? The experiment with higher resolution (atmosphere: T63L 31, ocean: $1.5^{\circ} \times 1.5^{\circ}$ ) includes small-scale processes beyond the truncation scale of T31. In this experiment nonlinear scale-interactions across this scale are possible. In Figures $1 j-11$ the variability of the two different model resolutions are compared. When comparing Figures $1 \mathrm{~d}-1 \mathrm{i}$ with Figures $1 \mathrm{j}-11$ some similarities but also clear differences can be observed. The increased model resolution leads to enhanced small-scale variability of vorticity and divergence (similar to our idealized experiments). However, the temperature pattern differs. Spectral coefficients on smallest scales show a slightly higher variability. Except for the total wavenumbers $l=31$, higher variability is mostly found for large zonal wavenumbers $m$, independent of $l$. There are also spectral coefficients, in particular those 
Table 2. Global Means and Responses to a $\mathrm{CO}_{2}$ Doubling for Temperatures at the Surface, $\left\langle T_{\text {surf }}\right\rangle$, and at $300 \mathrm{hPa},\langle T 300\rangle$ in $[\mathrm{K}]$, Based on 50-Year Averages

\begin{tabular}{lccccc}
\hline & \multicolumn{2}{c}{$\left\langle T_{\text {surf }}\right\rangle$, in $[\mathrm{K}]$} & & \multicolumn{2}{c}{$\langle T 300\rangle$, in $[\mathrm{K}]$} \\
\cline { 2 - 3 } \cline { 5 - 6 } Experiment & $1 \mathrm{xCO}_{2}$ & $2 \mathrm{xCO}_{2}-1 \mathrm{xCO}_{2}$ & & $1 \mathrm{xCO}_{2}$ & $2 \mathrm{xCO}_{2}-1 \mathrm{xCO}_{2}$ \\
\hline ctrl & 288.0 & 3.8 & & 232.2 & 5.6 \\
diffus_24 & 288.2 & 4.1 & & 232.6 & 6.1 \\
diffus_36 & 288.3 & 4.3 & & 232.8 & 6.4 \\
noise_3 & 287.9 & 3.9 & & 230.8 & 5.1 \\
noise_6 & 285.9 & 3.3 & 230.1 & 3.6 \\
\hline
\end{tabular}

with small zonal wavenumbers and large total wavenumbers, which display less variability.

[15] The climate sensitivities (i.e., the changes of the global mean surface temperatures due to doubled $\mathrm{CO}_{2}$ concentration) for all pairs of experiments are given in Table 2. In the control integrations the climate sensitivity is $3.8 \mathrm{~K}$. This value increases to $4.3 \mathrm{~K}$ if the horizontal diffusion is reduced by a factor of three. The climate sensitivity in the noise experiments with moderate noise intensity does not significantly change, but it decreases to $3.3 \mathrm{~K}$ in noise $2 \mathrm{x} \_6$ - noise $1 \mathrm{x} \_6$.

[16] The influence of the enhanced small-scale fluctuations is not confined to the surface response. Rather, it increases with height and reaches the largest values in the upper troposphere (Figure 2). Reducing the horizontal diffusion by a factor of 3 leads to a maximum amplification of the temperature response at $250 \mathrm{hPa}$ from $5.5 \mathrm{~K}$ to $6.4 \mathrm{~K}$ $(16 \%)$. However, if white noise is added to the small-scale spectral coefficients, the temperature response in the troposphere is decreased. The warming in the experiments with high noise intensity (noise1 1 6 and noise $2 \times 6$ ) is much smaller than the warming in the control experiments. In the experiments noise $1 x \_3$ and noise $2 x \_3$ the temperature response decreases only in the high troposphere with a maximum reduction at $200 \mathrm{hPa}$ from $4.8 \mathrm{~K}$ to $4.1 \mathrm{~K}(15 \%)$. The changes in mean temperature responses are statistical significant in all tropospheric levels except for the vertical range of $1000 \mathrm{hPa}$ to $450 \mathrm{hPa}$ of noise $2 \mathrm{x} \_3$ - noise1x_3.

[17] When enhancing the small-scale fluctuations not only the sensitivity of the temperature is changed but also the absolute mean temperature values of the $1 \times \mathrm{CO}_{2}-$ experiments. This is most pronounced in the noise experiments. The mean temperature at $300 \mathrm{hPa}$ drops, for example, from $232.2 \mathrm{~K}$ in ctrll $\mathrm{x}$ to $230.8 \mathrm{~K}$ in noise1x_3 (Table 2). Apart from the temperature also other climate variables are changed noticeable in the $1 \times \mathrm{CO}_{2}-$ noiseexperiments. In experiment noise $1 x_{-} 3$ we observe, for example, decreased zonal winds, a weakened atmospheric meridional circulation and less clouds (not shown). The changes of the general circulation could be explained by the 'tail wagging the dog' effect [Frederiksen et al., 2003]. In experiment noise1x_6 these changes are enhanced and lead to a rather unrealistic climate.

\section{Discussion and Conclusions}

\subsection{Differences of the Two Approaches}

[18] At first sight it is surprising that the climate sensitivity increases when using a reduced horizontal diffusion but it tends to decrease when adding noise to the small-scale components. Both methods enhance the small-scale variability and should therefore have a similar effect on the system. However, when having a closer look at the two methods, it becomes clear that they change the small-scale spectral coefficients in very different ways. The reduced horizontal diffusion results in less damping of small-scale eddies. Therefore, small-scale eddies are stronger and can interact more effectively with large-scale processes. These intensified small-scale eddies are correlated in time and across wavenumbers, and they are consistent with the governing equations of the model. In contrast, adding white noise to the small-scale components artificially perturbs the model. The resulting fluctuations are uncorrelated in time and spectral space. This could be appropriate, if the scales of the resolved variability were clearly separated from those of the dominant unresolved scales.

[19] Apart from the factors discussed above, the changes in the mean climate may also play a role. While in diffus $1 x$ 36 the mean climate is not very different in comparison to ctrllx, it has changed in noise $1 x \_3$. Owing to the new climate in noise1x_3, the thermodynamical feedback processes (e.g., cloud feedbacks) are altered. In this case, the climate sensitivity can be changed both due to nonlinear dynamical and thermodynamical processes. Small-scale variability can therefore not only have a direct effect on the model sensitivity but also indirect effects via influencing the thermodynamical feedback processes. In general it is hard to distinguish between direct and indirect effects of enhanced small-scale fluctuations on the climate sensitivity. Nevertheless, the noise experiments might be affected more by indirect effects than the runs with reduced horizontal diffusion.

\subsection{Validity of Horizontal Diffusion}

[20] The results shown in Figure 2 suggest that a reduction of the horizontal diffusion leads to a higher climate sensitivity. The question arising is how well established is the strength and structure of the horizontal diffusion. Several studies [e.g., Koshyk and Boer, 1995; Kaas et al., 1999; Frederiksen and Kepert, 2006] sought to improve the

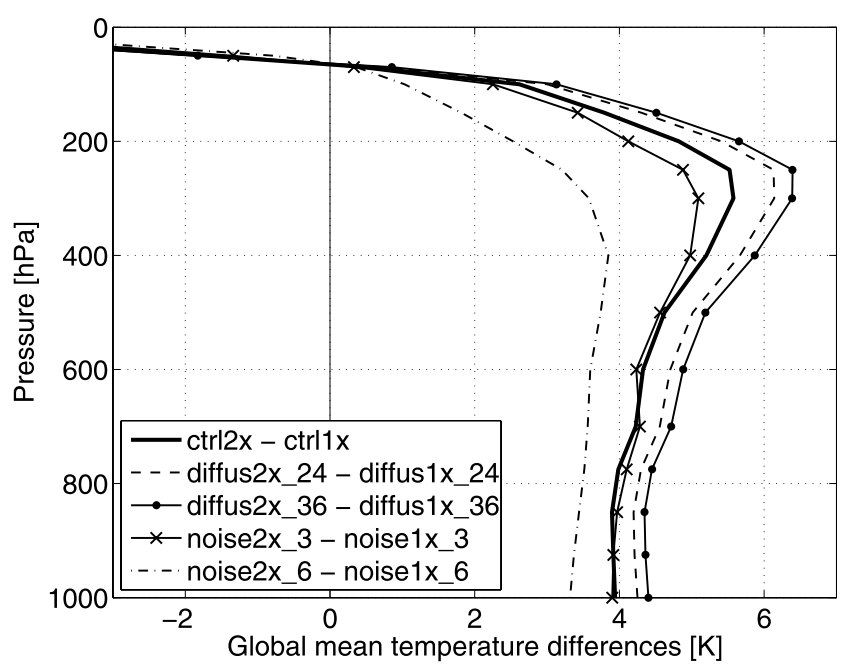

Figure 2. Climate response of temperature to $\mathrm{CO}_{2}$ forcing obtained from experiments with different representations of small scale fluctuations. 
parameterization of the nonlinear interactions between subgrid scales and resolved scales. Besides confirming the idea of damping high wavenumbers stronger than lower wavenumbers, they found also a 'negative damping' at intermediate wavenumbers. This suggests that certain scales are enhanced instead of damped through the scale interactions with subgrid scales. The damping strength of the horizontal diffusion in climate models is generally tuned to ensure a kinetic energy spectrum close to observations. But, as mentioned by Stephenson [1995], why should the kinetic energy spectrum of a discrete model (especially near the truncation scale) look alike the spectrum of a continuous system? Enhanced small-scale energy might even be necessary to simulate the large-scale circulation and future climate changes correctly. Given the uncertainty of the form and strength of the horizontal diffusion in climate models, our results suggest that the modeled climate sensitivity to $\mathrm{CO}_{2}$ forcing does not only depend on parameterizations related to thermodynamical feedback processes but also on the parameterization of nonlinear interactions between dynamical subgrid-scale processes and resolved scales.

[21] Acknowledgments. We thank Johann Jungclaus and Erich Roeckner for useful discussions. We thank Jorgen Frederiksen and one anonymous reviewer for their constructive comments and suggestions. This work was supported by the Max Planck Society and the International Max Planck Research School on Earth System Modelling.

\section{References}

Bony, S., et al. (2006), How well do we understand and evaluate climate change feedback processes?, J. Clim., 19(15), 3445-3482.

Colman, R. (2003), A comparison of climate feedbacks in general circulation models, Clim. Dyn., 20(7-8), 865-873.

Frederiksen, J. S., and S. M. Kepert (2006), Dynamical subgrid-scale parameterizations from direct numerical simulations, J. Atmos. Sci., 63(11), $3006-3019$

Frederiksen, J. S., M. R. Dix, and A. G. Davies (2003), The effects of closure-based eddy diffusion on the climate and spectra of a GCM, Tellus, $55(1), 31-44$.

Intergovernmental Panel on Climate Change (IPCC) (2007), Climate Change 2007: The Physical Science Basis. Contribution of Working Group I to the Fourth Assessment Report of the Intergovernmental Panel on Climate Change, Cambridge Univ. Press, Cambridge, U. K.

Jungclaus, J. H., N. Keenlyside, M. Botzet, H. Haak, J. J. Luo, M. Latif, J. Marotzke, U. Mikolajewicz, and E. Roeckner (2006), Ocean circula- tion and tropical variability in the coupled model ECHAM5/MPI-OM, J. Clim., 19(16), 3952-3972.

Kaas, E., A. Guldberg, W. May, and M. Deque (1999), Using tendency errors to tune the parameterisation of unresolved dynamical scale interactions in atmospheric general circulation models, Tellus, 51(5), 612629.

Kiehl, J. T., C. A. Shields, J. J. Hack, and W. D. Collins (2006), The climate sensitivity of the Community Climate System Model version 3 (CCSM3), J. Clim., 19(11), 2584-2596.

Knutti, R., G. A. Meehl, M. R. Allen, and D. A. Stainforth (2006), Constraining climate sensitivity from the seasonal cycle in surface temperature, J. Clim., 19(17), 4224-4233.

Koshyk, J., and G. Boer (1995), Parameterization of dynamical subgridscale processes in a spectral GCM, J. Atmos. Sci., 52(7), 965-976.

Leith, C. (1975), Climate response and fluctuation dissipation, J. Atmos. Sci, 32, 2022-2026.

Marsland, S. J., H. Haak, J. H. Jungclaus, M. Latif, and F. Roske (2003), The Max-Planck-Institute global ocean/sea ice model with orthogonal curvilinear coordinates, Ocean Modell., 5(2), 91-127.

May, W., and E. Roeckner (2001), A time-slice experiment with the ECHAM4 AGCM at high resolution: The impact of horizontal resolution on annual mean climate change, Clim. Dyn., 17(5-6), 407-420.

Murphy, J. M., D. M. H. Sexton, D. N. Barnett, G. S. Jones, M. J. Webb, and M. Collins (2004), Quantification of modelling uncertainties in a large ensemble of climate change simulations, Nature, 430(7001), $768-772$.

Roeckner, E., et al. (2003), The atmospheric general circulation model ECHAM5: Part I. Model description, Tech. Rep. 349, Max Planck Inst. for Meteorol., Hamburg, Germany.

Roeckner, E., R. Brokopf, M. Esch, M. Giorgetta, S. Hagemann, L. Kornblueh, E. Manzini, U. Schlese, and U. Schulzweida (2006), Sensitivity of simulated climate to horizontal and vertical resolution in the ECHAM5 atmosphere model, J. Clim., 19(16), 3771-3791.

Seiffert, R., R. Blender, and K. Fraedrich (2006), Subscale forcing in a global atmospheric circulation model and stochastic parametrization, O. J. R. Meteorol. Soc., 132(618), 1627-1643.

Soden, B. J., and I. M. Held (2006), An assessment of climate feedbacks in coupled ocean-atmosphere models, J. Clim., 19(14), 3354-3360.

Stephenson, D. B. (1995), The impact of changing the horizontal diffusion scheme on the northern winter climatology of a general circulation model, Q. J. R. Meteorol. Soc., 121(521), 211-226.

von Storch, J. S. (2004), On statistical dissipation in GCM-climate, Clim. Dyn., 23(1), 1-15.

Webb, M. J., et al. (2006), On the contribution of local feedback mechanisms to the range of climate sensitivity in two GCM ensembles, Clim. Dyn., 27(1), 17-38.

R. Seiffert and J.-S. von Storch, Max Planck Institute for Meteorology, Bundesstr. 53, D-20146 Hamburg, Germany. (rita.seiffert@ zmaw.de; jin-song.von.storch@zmaw.de) 